\title{
Spinal Cord Transection in a Type II Odontoid Fracture From a Ground-Level Fall
}

\author{
Samiat Agunbiade ${ }^{1}$, Patrick J. Belton ${ }^{1}$, Fassil B. Mesfin ${ }^{1}$ \\ 1. Neurosurgery, University of Missouri School of Medicine, Columbia, USA
}

Corresponding author: Samiat Agunbiade, samiat.agunbiade@gmail.com

\begin{abstract}
Odontoid fractures typically occur as a result of trauma: high-velocity injuries like motor vehicle accidents in young people and falls for the elderly. Odontoid fractures are the most common cervical spine fractures in patients over 65 , with type II being the most common. However, spinal cord transections are rare with these types of injuries, especially without significant fracture displacement, translation or evidence of ligamentous disruption on post-injury imaging. We report a case of a patient who sustained a spinal cord transection secondary to an acute type II odontoid fracture from a ground-level fall, without computed tomography radiographic evidence of cord disruption or impingement.
\end{abstract}

Categories: Neurosurgery

Keywords: odontoid fracture, spinal cord transection, ground level fall

\section{Introduction}

Odontoid fractures account for 10-15\% of cervical spine fractures, with type II (fracture through the base of the dens) being the most common, accounting for over $50 \%$, followed by type III (fracture through the body of the dens), and type I (fracture of the tip of the dens) being exceedingly rare [1,2]. Odontoid fractures typically result from blunt trauma to the cervical spine, most commonly high-velocity injuries in younger patients, such as motor vehicle accidents, and falls in elderly patients [2,3]. Odontoid fractures are the most common cervical spine fractures in patients over 65 with type II the being most common $[4,5]$. Although odontoid fractures have a neurological injury rate ranging from $7.5 \%$ to $33 \%$ [5-7], cervical spinal cord injuries following odontoid fractures are rare [6], especially without significant translation, displacement or ligamentous disruption. This is likely to the relatively large cross-sectional diameter of the spinal canal at that odontoid level, compared to the diameter of the spinal cord [1]. We report a case of a patient who sustained a spinal cord transection following a type II odontoid fracture from a ground-level fall.

Review began 11/03/2020 Review ended 12/22/2020 Published 12/28/2020

\section{Copyright 2020}

Agunbiade et al. This is an open access article distributed under the terms of the Creative Commons Attribution License CC-BY 4.0., which permits unrestricted use, distribution, and reproduction in any medium, provided the original author and source are credited.

\section{Case Presentation}

The patient was a 78-year-old female with a past medical history of type II diabetes mellitus who was found unresponsive by her family following a ground-level fall. The patient was carrying groceries and collapsed or tripped, striking her face. The event was witnessed by one of her daughters. Her daughter reported that on rushing to her assistance she had no pulse and had turned blue. Bystanders performed cardiopulmonary resuscitation (CPR) while waiting for medical assistance. When emergency medical personnel arrived, the patient was opening her eyes but did not respond to any external stimuli and was subsequently was intubated. When the patient arrived at the outside hospital, she was hypotensive with a blood pressures of 60/40 and Philadelphia type cervical collar was placed. The patient was transferred to a tertiary care center for a higher level of care.

On arrival at the tertiary care center, Glasgow Coma Score was 3(t). Pupils were equal and reactive to light, but sluggish, with random eye movements. She had no corneal reflex or cough reflexes, but a mild gag reflex was appreciated. There were periodic facial movements that resembled myoclonic jerks. No rectal tone was noted, but diffuse hyperreflexia was noted. Computed tomography (CT) of the brain was negative for intracranial hemorrhages, but CT of the cervical spine demonstrated a C1 posterior arch fracture (Figure 1), and acute mildly displaced type II odontoid fracture with $2-3 \mathrm{~mm}$ posterior displacement and mild posterior subluxation of C1 on C2 (Figures 2 and 3). Whole-body survey imaging was significant for multiple rib fractures, likely from CPR in the field. 


\section{Cureus}

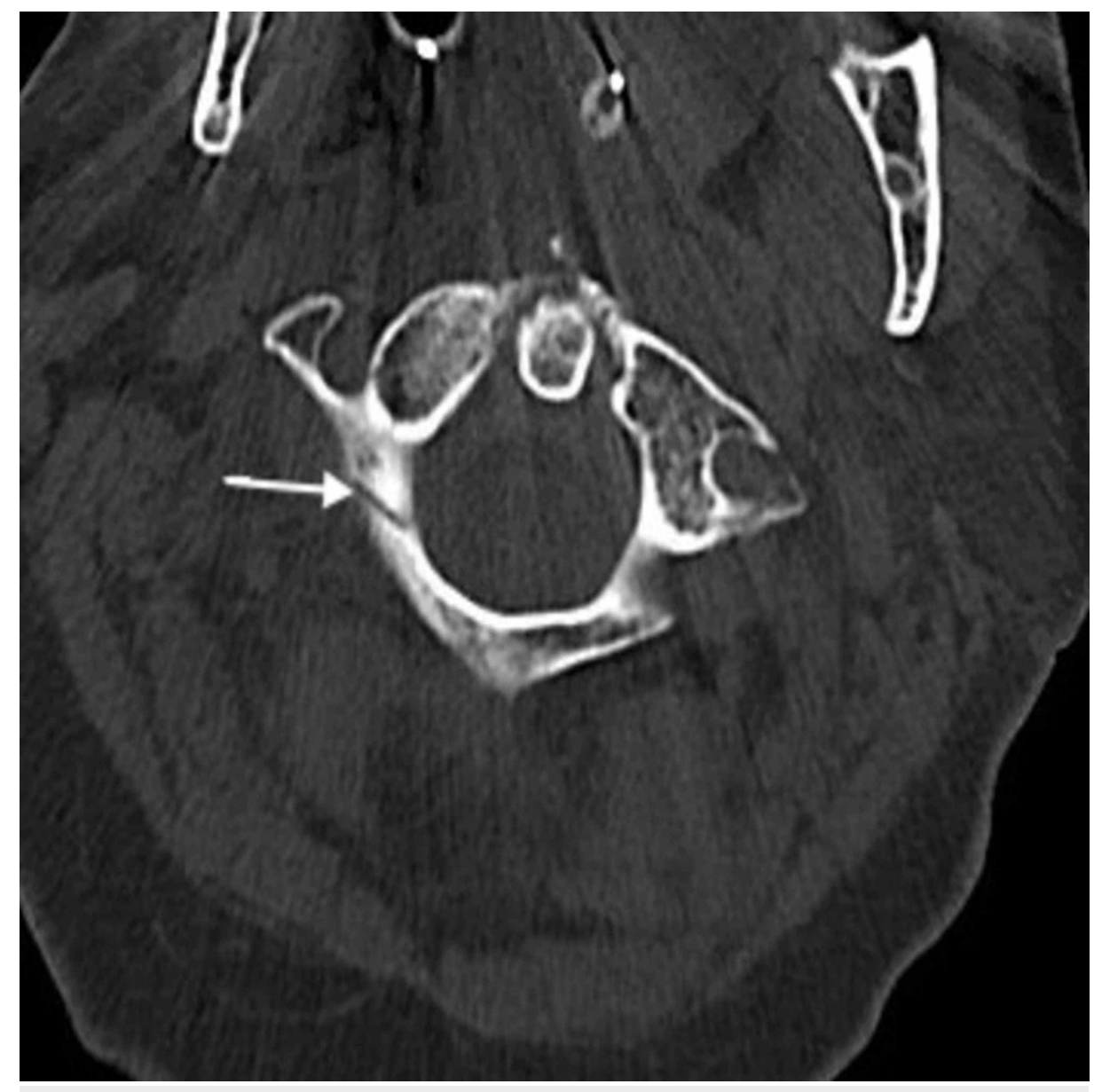

FIGURE 1: Axial CT of the cervical spine showing C1 posterior arch fracture.

There is an acute non-displaced $\mathrm{C} 1$ arch fracture with bilateral transverse foramen involvement without displacement. 


\section{Cureus}

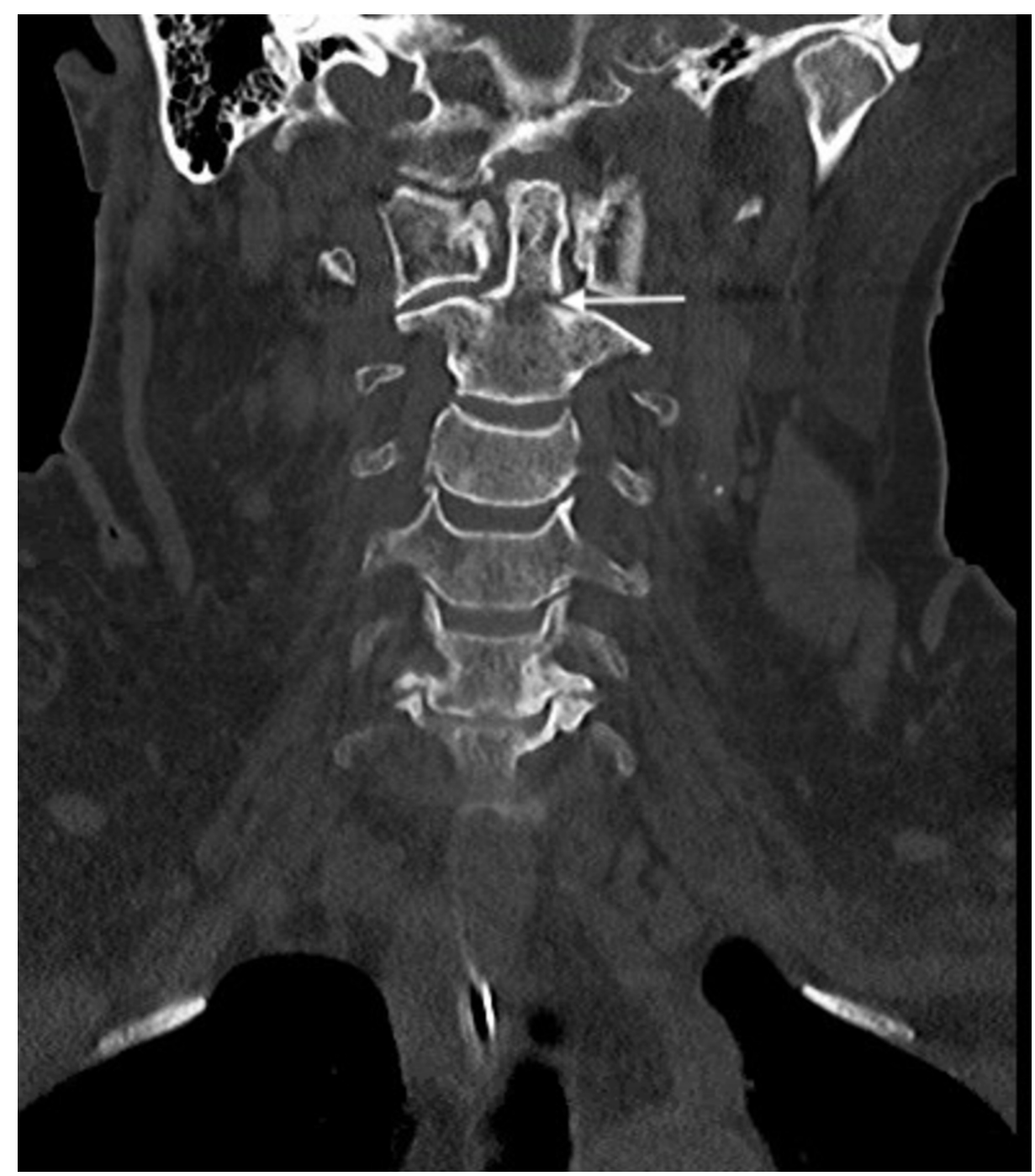

FIGURE 2: Coronal CT of the cervical spine with type II odontoid fracture.

Coronal non-contrast CT bone window of type II odontoid fracture. 


\section{Cureus}

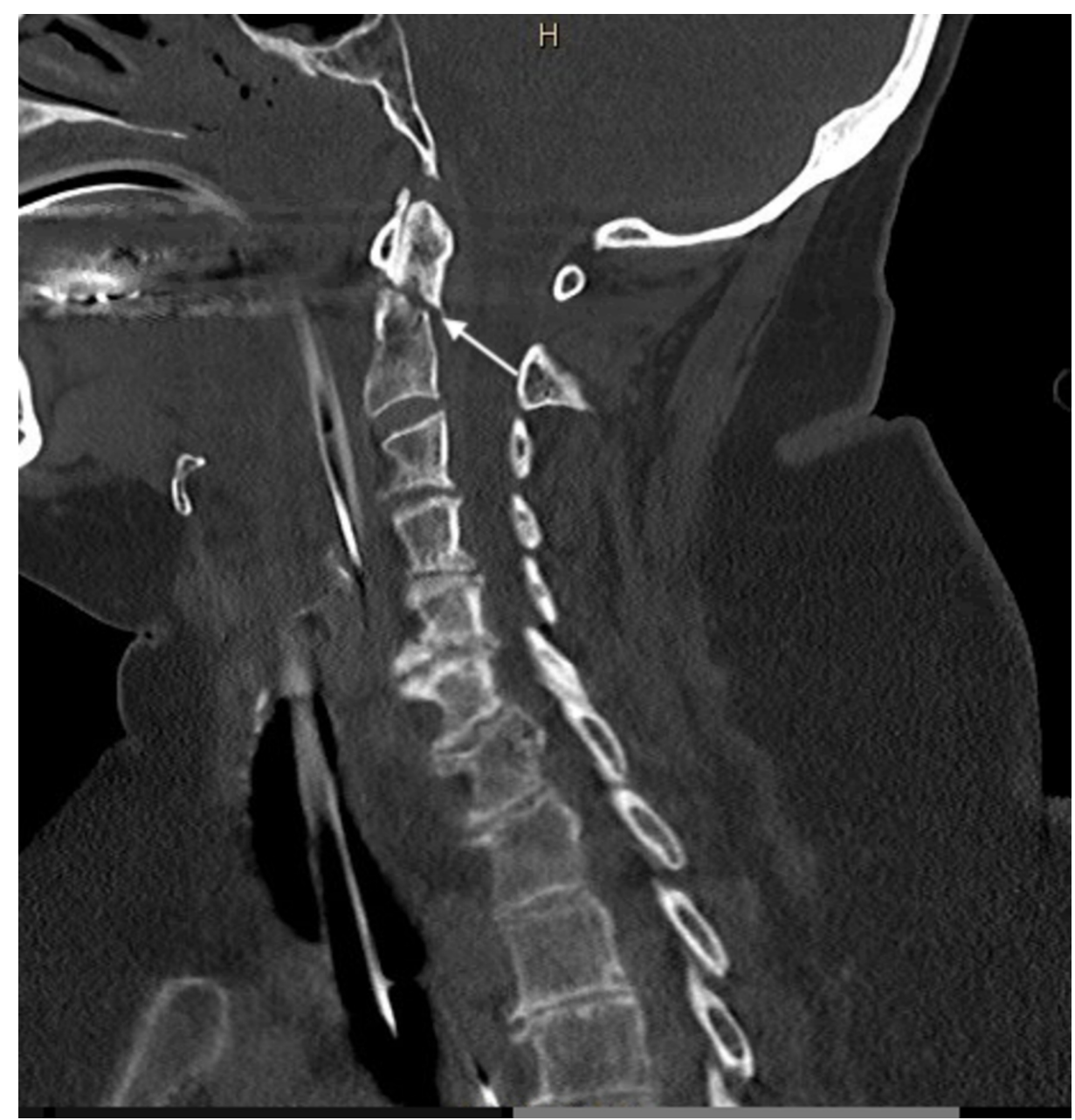

\section{FIGURE 3: Parasagittal section of type II odontoid fracture.}

The parasagittal view of the odontoid fracture shows 2-3 mm posterior displacement and subluxation of $\mathrm{C1}$ on C2.

The patient was placed in a Miami J collar and admitted to the intensive care unit for suspected anoxic brain injury. There was concern that the patient had experienced a cardiac event that caused a sudden loss of pulse. However, multiple troponins were negative and the admission electrocardiogram did not show ST elevations consistent with a cardiac event. Doppler studies showed an occlusive deep vein thrombosis in the right peroneal vein. A saddle pulmonary embolism was suspected to have caused a syncopal episode and cardiac arrest; the patient was started on heparin. Echocardiogram showed normal left and right ventricular size, with an ejection fraction of $55 \%$ and normal wall motion throughout. CT of the chest with pulmonary embolism protocol did not demonstrate a pulmonary embolism. An electroencephalogram was performed, and the results were consistent with severe diffuse encephalopathy, with no signs of seizure activity.

Magnetic resonance imaging (MRI) of the brain and cervical spine were performed. On the MRI of the cervical spine, the patient was found to have a transection of the spinal cord at C1-C2 (Figure 4). Short tau inversion recovery (STIR) sequence redemonstrated the acute spinal injury without ligamentous disruption. 


\section{Cureus}

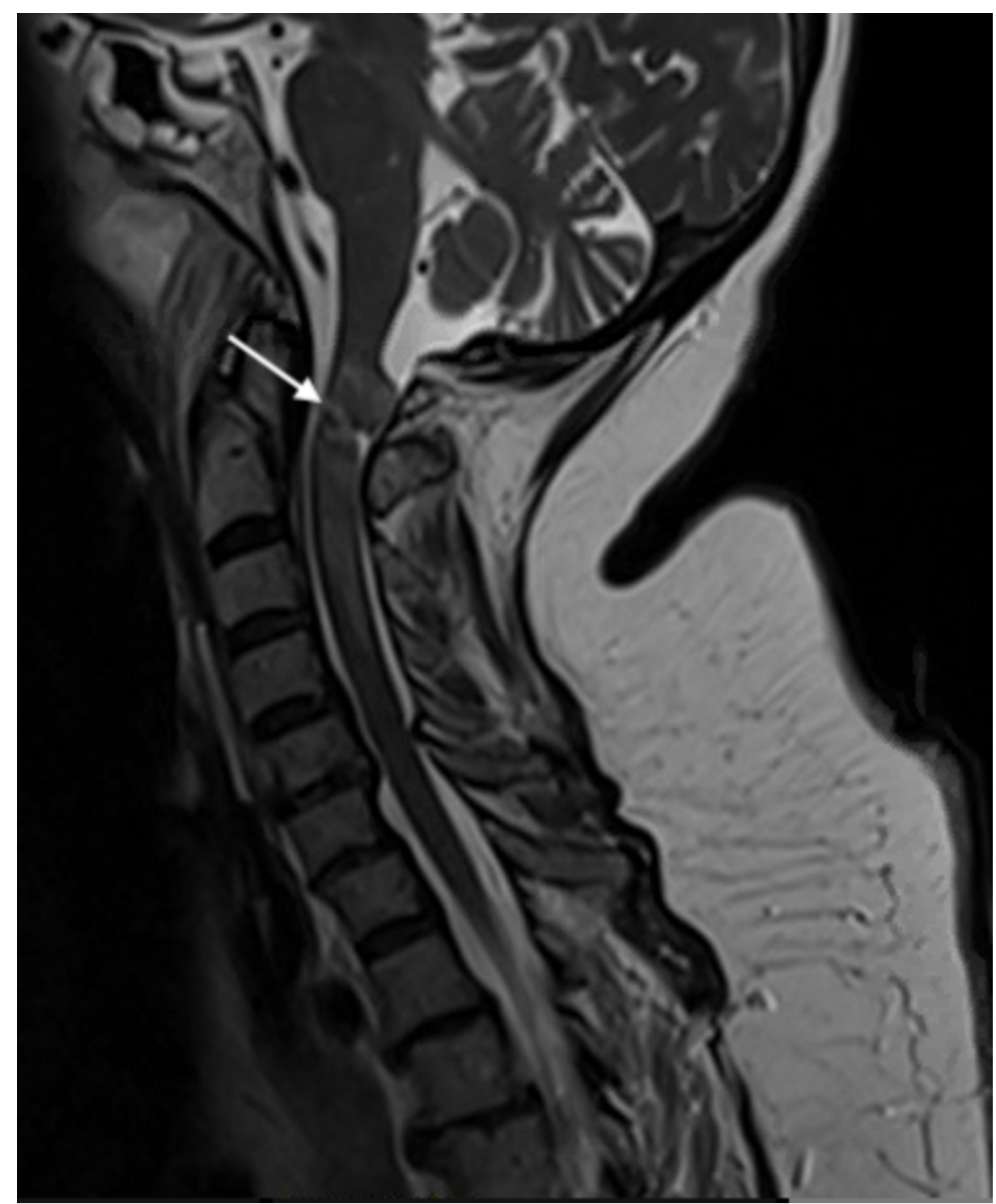

FIGURE 4: T2-weighted MRI of the cervical spine with a parasagittal view.

The parasagittal view demonstrates high-grade partial spinal cord transection at the level of C1-C2. There is also an associated spinal cord contusion and a small amount of intrathecal blood.

The patient exhibited signs of neurogenic shock, with bradycardia, hypotension, and warm extremities. The MRI results, prognosis, and the likely possibility of locked in phenomenon were discussed with the family. Ultimately the family rescinded a do not resuscitate order and elected a palliative route. The patient was extubated and passed away soon after.

\section{Discussion}

Odontoid fractures are more commonly seen in higher velocity impacts, and although odontoid fractures in the elderly are not rare, an injury this severe, given the mechanism of a ground-level fall, is extremely unusual. For the elderly population, the odontoid fractures can occur from low energy impacts such as falls from standing positions, likely due to decreased bone density; with hyperextension of the C-spine, causing the head of $\mathrm{C} 1$ to be pushed backwards being the most common mechanism of injury [6-8]. Hyperflexion can also lead to odontoid fractures due to the transmission of excess forces via the transverse ligament [2]. Given the facial injury, hyperextension may have been the patient's mechanism of injury. There may also be transverse ligament tears associated with type II fractures. Many type II fractures can mimic transverse ligament injuries with the loss of translational restriction of $\mathrm{C} 1$ on $\mathrm{C} 2$, increasing the risk of spinal cord injury and cranio-cervical deformities with poor healing [8]. However, in our patient, on MRI, there was no indication of significant ligamentous damage and displacement in her MRI. There was no indication of spinal cord compression, distraction injury, or significantly large hematoma that may have contributed to 
the spinal cord transection in this patient.

Traumatic cervical fractures with translation and displacement are unstable and are at increased risk of spinal cord injury [9]. However, in this patient, there was no significant displacement of the C2 fracture on survey imaging in the rigid collar, which would make the spinal cord transection that occurred in this patient even more extraordinary. The translation is defined as $3.5 \mathrm{~mm}$ or more of sagittal or coronal malalignment of one or more cervical vertebrae [10,11]. There have been no previously reported cases of spinal cord injuries in non-displaced fractures. The $\mathrm{C} 1$ fracture was nondisplaced and the odontoid fracture sustained by our patient had only a mild posterior displacement of 2-3 mm, and mild posterior subluxation of C1 on C2. We suspect the patient has had a transient displacement of the fracture, causing the spinal cord transection. Additionally, we hypothesize that the patient may have also suffered an anoxic brain as the result of the high spinal cord transection, given the myoclonic facial jerks. The C2 spinal cord transection likely led to phrenic nerve paralysis since phrenic nerve innervation occurs via C3-5 causing diaphragm paralysis, rendering the patient unable to breathe on her own and contributing to an anoxic brain injury. The patient's hypotension was likely due to neurogenic shock from the high spinal cord injury further exacerbating her anoxic brain injury. Neurogenic shock can be seen in spinal cord injuries above T6 and is characterized by hypotension, bradycardia and autonomic dysfunction [12]. Hypotension occurs as the result of the loss of systemic vascular resistance from the loss of sympathetic tone, leading to vasodilation from unopposed parasympathetic responses [12]. Flexion force may result in airway edema, resulting in airway obstruction [13]. The patient had a normal echocardiogram and imaging did not demonstrate any signs of a pulmonary embolism, ruling out a cardiac source of hypotension. The patient was likely exhibiting signs of a locked-in syndrome as the result of her injuries.

Although spinal cord injuries following odontoid fractures are rare, there are certain factors that increase the risk of sustaining spinal cord injuries. Harrop et al. found that spinal cord injuries from odontoid fractures are more typically seen in males, with smaller spinal canals that suffer from high-velocity injuries [6]. Degenerative changes of the spinal cord such as spondylosis and ossification of the posterior longitudinal ligament and more narrow spinal canals are important preexisting factors to the risk of sustaining spinal cord injuries [14].

Many in the elderly population are at higher risk of surgical complications due to co-morbidities or poor bone quality but type II odontoid fractures are considered inherently unstable and are at increased risk of nonunion due to poor vascularity of the odontoid process [8]. There are high rates of nonunion with conservative management in elderly patients $[1,5,8]$; patients over 50 have a 21 -fold greater incidence of nonunion with surgical management [15]. Chapman et al. in their study of 322 patients over 65 with type II odontoid fractures found that surgical treatment did not negatively impact survival; in fact, their study showed an improvement in 30-day survival and improved long-term survival with the surgical intervention [16]. Typical surgical strategies for type II odontoid fractures include direct anterior odontoid screw fixation, and posterior cervical instrumental fusion $[8,17]$. Nonsurgical management consists of rigid and non-rigid immobilizations with a halo or cervical collar $[5,15]$. The integrity of the transverse and alar ligaments also plays a significant role in determining surgical versus nonsurgical treatment, as well as the degree of anterior-posterior displacement $(>5 \mathrm{~mm})$ and angulation $\left(>11^{\circ}\right)[7,9,15]$.

\section{Conclusions}

Odontoid fractures are the most common cervical spine fractures in the elderly. Severe spinal cord injuries from odontoid fractures are rare but can occur, even without significant disruption of the supporting ligaments. Spinal cord injuries should always be considered in patients with odontoid fractures. MRI imaging for spinal cord injury and ligamentous disruption should be considered in all patients with traumatic odontoid fractures and significant neurologic findings.

\section{Additional Information \\ Disclosures}

Human subjects: Consent was obtained by all participants in this study. Conflicts of interest: In compliance with the ICMJE uniform disclosure form, all authors declare the following: Payment/services info: All authors have declared that no financial support was received from any organization for the submitted work. Financial relationships: All authors have declared that they have no financial relationships at present or within the previous three years with any organizations that might have an interest in the submitted work. Other relationships: All authors have declared that there are no other relationships or activities that could appear to have influenced the submitted work.

\section{References}

1. Anderson LD, D'Alonzo RT: Fractures of the odontoid process of the axis . J Bone Joint Surg Am. 1974, 56:1663-1674. 10.2106/00004623-197456080-00017

2. Robinson AL, Möller A, Robinson Y, Olerud C: C2 fracture subtypes, incidence, and treatment allocation change with age: a retrospective cohort study of 233 consecutive cases. Biomed Res Int. 2017, 2017:8321680. $10.1155 / 2017 / 8321680$ 
3. Al-Mahfoudh R, Beagrie C, Woolley E, et al.: Management of typical and atypical Hangman's fractures . Global Spine J. 2016, 6:248-256. 10.1055/s-0035-1563404

4. Lomoschitz FM, Blackmore CC, Mirza SK, Mann FA: Cervical spine injuries in patients 65 years old and older: epidemiologic analysis regarding the effects of age and injury mechanism on distribution, type, and stability of injuries. AJR Am J Roentgenol. 2002, 178:573-577. 10.2214/ajr.178.3.1780573

5. Iyer S, Hurlbert RJ, Albert TJ: Management of odontoid fractures in the elderly: a review of the literature and an evidence-based treatment algorithm. Neurosurgery. 2018, 82:419-430. 10.1093/neuros/nyx546

6. Harrop JS, Sharan AD, Przybylski GJ: Epidemiology of spinal cord injury after acute odontoid fractures . Neurosurg Focus. 2000, 8:e4.

7. Müller EJ, Schwinnen I, Fischer K, Wick M, Muhr G: Non-rigid immobilisation of odontoid fractures . Eur Spine J. 2003, 12:522-525. 10.1007/s00586-003-0531-1

8. Saro A, Abdelhameid AK, Fadl KN: Surgical outcome of type II odontoid fracture, Harms technique . Egypt J Neurosurg. 2019, 34:3. 10.1186/s41984-019-0031-1

9. White AA 3rd, Johnson RM, Panjabi MM, Southwick WO: Biomechanical analysis of clinical stability in the cervical spine. Clin Orthop Relat Res. 1975, 109:85-96. 10.1097/00003086-197506000-00011

10. Dvorak M, Wing P, Fehlings M, Vaccaro AR: International spinal cord injury spinal column injury basic data set. Spinal Cord. 2012, 50:817-821. 10.1038/sc.2012.60

11. White AA, Panjabi MM: Clinical Biomechanics of the Spine. JB Lippincott, Philadelphia; 1990.

12. Stein DM, Knight WA 4th: Emergency neurological life support: traumatic spine injury. Neurocrit Care. 2017, 27:170-180. 10.1007/s12028-017-0462-z

13. Jabbour P, Fehlings M, Vaccaro AR, Harrop JS: Traumatic spine injuries in the geriatric population. Neurosurg Focus. 2008, 25:E16. 10.3171/foc.2008.25.11.e16

14. Koyanagi I, Iwasaki Y, Hida K, Akino M, Imamura H, Abe H: Acute cervical cord injury without fracture or dislocation of the spinal column. J Neurosurg. 2000, 93:15-20. 10.3171/spi.2000.93.1.0015

15. Young CC, Chiarelli PA, Hofstetter CP: Injuries to the cervical spine. Principles of Neurological Surgery. Ellenbogen RG, Sekhar LN, Kitchen ND, Brito da Silva H (ed): Elsevier, Amsterdam; 2018. 475-492.

16. Chapman J, Smith JS, Kopjar B, Vaccaro AR, Arnold P, Shaffrey CI, Fehlings MG: The AOSpine North America Geriatric Odontoid Fracture Mortality Study: a retrospective review of mortality outcomes for operative versus nonoperative treatment of 322 patients with long-term follow-up. Spine (Phila Pa 1976). 2013, 38:1098-1104. 10.1097/BRS.0b013e318286f0cf

17. Apfelbaum RI, Lonser RR, Veres R, Casey A: Direct anterior screw fixation for recent and remote odontoid fractures. J Neurosurg. 2000, 93:227-236. 10.3171/spi.2000.93.2.0227 